\title{
Generalized homogeneous approximations and the global stabilization problem of nonlinear systems
}

\author{
Stefano Battilotti
}

\begin{abstract}
We introduce a new notion of generalized homogeneous approximation at the origin and at infinity which extends the classical notions. Homogeneous systems in the generalized sense are a very large class of nonlinear systems, including (lower and upper) triangular systems. Exploiting this extension we give basic results concerning stabilization and robustness of nonlinear systems which have a generalized homogeneous approximation at zero and at the origin.
\end{abstract}

\section{INTRODUCTION}

The problem of designing stabilizing feedback control laws for nonlinear systems has been addressed by many authors with different approaches. Many of these use domination tools and robustness. In a domination approach the stability of a system $\dot{x}=f(x)+g(x) u+\phi(x)$ is ensured by designing a stabilizing feedback controller for $\dot{x}=f(x)+$ $g(x) u$ provided that the stability property of the closed-loop system is robust with respect to the perturbation $\phi(x)$. This domination idea has been largely exploited by employing homogeneous feedback controllers with homogeneous systems $\dot{x}=f(x)+g(x) u$ ([4], [11], [5], [8], [9], [10]). The idea of extending this approach to system which are not homogeneous but admit a homogeneous approximation at the origin, i.e. when the state tends to the origin, is pursued in [3], [11] and [5]. Recently, in [2] this domination technique has been extended to systems which are not homogenous but become homogeneous as the state tends to the origin or to infinity but with different weights and degrees (homogeneous approximation in the bi-limit). However, maps like $f(x)=$ $\left(x_{2}, c_{0} x_{2}^{q}+c_{\infty} x_{2}^{p}\right)^{T}, 0<q<p$, admits a homogeneous approximation at the origin and at infinity only when $p<2$. Homogeneity (in the bi-limit) in the classical sense of a given map $f(x)$ is characterized by some degree $\mathfrak{d}$ and weights $\mathfrak{r}$. In this paper, following some preliminary papers ([1]), we introduce a generalized notion of homogeneity (in the bi-limit), which significantly enlarges the class of homogeneous maps (in the classical sense). The difference is that a generalized homogeneous map $f(x)$ is characterized by some vector degree $\mathfrak{d}$ and weights $\mathfrak{r}$. The degree $\mathfrak{d}_{i}$ of $f(x)$ is the homogeneity degree of the component $f_{i}(x)$. For example, $f(x)=\left(x_{2}, c_{0} x_{2}^{q}+c_{\infty} x_{2}^{p}\right)^{T}, 0<q<p$, is homogeneous (in the bi-limit) in the generalized sense whatever $p>q$ is. The homogeneity in a generalized sense (unlike the notion of generalized homogeneity introduced in [1]) shares all its properties with the homogeneity in the classical sense ([4], [11], [5], [8], [2]). We will use this new

S. Battilotti is with the Dipartimento di Ingegneria Informatica, Automatica e Gestionale "Antonio Ruberti", Università di Roma "La Sapienza" battilottiedis.uniromal.it notion to design a homogeneous (in the bi-limit) stabilizing state-feedback together with a Lyapunov function with a homogeneous (in the bi-limit) derivative for a chain of power integrators and then consider this dynamics as the dominant dynamics near the origin and infinity of a chain of power integrators with nonlinear perturbations. By the domination approach we establish the global asymptotic stability of chain of power integrators with nonlinear perturbations. For reasons of space, we focus our analysis on state feedback stabilization, leaving output feedback stabilization for a more complete study.

\section{NOTATION}

- $\mathbb{R}^{n}$ (resp. $\mathbb{R}^{n \times n}$ ) is the set of $n$-dimensional real column vectors (resp. $n \times n$ matrices). $\mathbb{R}_{\geqslant}$(resp. $\mathbb{R}_{\geqslant}^{n}$ ) denotes the set of real non-negative numbers (resp. vectors in $\mathbb{R}^{n}$ with real non-negative entries) and $\mathbb{R}_{>}$(resp. $\mathbb{R}_{>}^{n}$ ) denotes the set of real positive numbers (resp. vectors in $\mathbb{R}^{n}$ with real positive entries).

- For any $G \in \mathbb{R}^{p \times n}$ we denote by $G_{i j}$ the $(i, j)$-th entry of $G$ and for any $G \in \mathbb{R}^{p}$ by $G_{i}$ the $i$-th element of $G$. We retain a similar notation for functions.

- For any real $\mathfrak{r}>0$ we define $w \mapsto w^{\mathfrak{r}}$ as $w^{\mathfrak{r}}=$ $\operatorname{sgn}\{w\}|w|^{\mathfrak{r}}$. Notice that

$$
w_{1}>w_{2} \Rightarrow w_{1}^{\mathfrak{r}}>w_{2}^{\mathfrak{r}}, \frac{d\left(w^{\mathfrak{r}}\right)}{d w}=\mathfrak{r}|w|^{\mathfrak{r}-1} .
$$

- The dilation of a vector $x \in \mathbb{R}^{n}$ with weights $\mathfrak{r} \in \mathbb{R}^{n}$ and parameter $\varepsilon>0$ is defined as

$$
\varepsilon^{\mathfrak{r}} \diamond x:=\left(\varepsilon^{\mathfrak{r}_{1}} x_{1}, \cdots, \varepsilon^{\mathfrak{r}_{n}} x_{n}\right)^{T}
$$

We also use the notation $x^{\mathfrak{p}}:=\left(x_{1}^{\mathfrak{p}_{1}}, \cdots, x_{n}^{\mathfrak{p}_{n}}\right)^{T}$ for $x, \mathfrak{p} \in \mathbb{R}^{n}$.

\section{GENERALIZED HOMOGENEITY}

In this section we introduce the notion of generalized homogeneity (in the bi-limit).

Definition 3.1: (Generalized homogeneity). A function $\phi \in \mathbf{C}^{0}\left(\mathbb{R}^{n}, \mathbb{R}^{l}\right)$ is said to be homogeneous (in the generalized sense) with triple $(\mathfrak{r}, \mathfrak{e}, \Phi)$ (weights $\mathfrak{r}$ and degrees $\mathfrak{e}$ ) if there exist $\mathfrak{e} \in \mathbb{R}_{\geqslant}^{l} \backslash\{0\}, \mathfrak{r} \in \mathbb{R}_{>}^{n}$ and $\Phi \in \mathbf{C}^{0}\left(\mathbb{R}^{n}, \mathbb{R}^{l}\right)$ such that for all $w \in \mathbb{R}^{n} \backslash\{0\}$ and $\varepsilon>0$

$$
\phi\left(\varepsilon^{\mathfrak{r}} \diamond w\right)=\varepsilon^{\mathfrak{e}} \diamond \Phi(w)
$$

Remark 3.1: Definition 3.1 generalizes the definition of homogeneity (in the classical sense: [4], [11], [5]) as follows: $\phi \in \mathbf{C}^{0}\left(\mathbb{R}^{n}, \mathbb{R}^{n}\right)$ is homogeneous (in the classical sense) with weights $\mathfrak{r}$ and degree $\mathfrak{e}_{0}$ if and only if $\phi$ is homogeneous 
(in the generalized sense) with weights $\mathfrak{r}$ and degrees $\mathfrak{e}$ such that $\mathfrak{e}_{j}-\mathfrak{r}_{j}=\mathfrak{e}_{0}$ for all $j=1, \ldots, n$.

Remark 3.2: Weigths and degrees are not uniquely defined. Any homogeneous (in the generalized sense) $\phi$ with triple $(\mathfrak{r}, \mathfrak{e}, \Phi)$ is also homogeneous (in the generalized sense) with triple $(k \mathfrak{r}, k \mathfrak{e}, \Phi)$ for any $k>0$.

The function

$$
\phi(x):=\left(\begin{array}{c}
x_{2} \\
x_{1}^{2}+x_{2}^{3}
\end{array}\right)
$$

is homogeneous (in the generalized sense) with triple

$$
(\mathfrak{r}, \mathfrak{e}, \Phi)=\left(\left(\begin{array}{c}
1 \\
\frac{2}{3}
\end{array}\right),\left(\begin{array}{c}
\frac{2}{3} \\
2
\end{array}\right),\left(\begin{array}{c}
x_{2} \\
x_{1}^{2}+x_{2}^{3}
\end{array}\right)\right)
$$

Note that $\phi$ is not homogeneous (in the classical sense).

If a function $\phi(x)$ is not homogeneous (in the generalized sense), it might become homogeneous (in the generalized sense) when $x$ is close to the origin or to infinity. The following definitions generalize the notion of homogeneous approximation of a function at infinity and around zero.

Definition 3.2: (Generalized homogeneity in the $\infty$ limit). A function $\phi \in \mathbf{C}^{0}\left(\mathbb{R}^{n}, \mathbb{R}^{l}\right)$ is said to be (in the generalized sense) homogeneous in the $\infty$-limit with triple $\left(\mathfrak{r}^{\infty}, \mathfrak{e}^{\infty}, \Phi^{\infty}\right)$ (weights $\mathfrak{r}^{\infty}$, degrees $\mathfrak{e}^{\infty}$ and approximating functions $\left.\Phi^{\infty}\right)$ if there exist $\mathfrak{e}^{\infty} \in \mathbb{R}_{\geqslant}^{l} \backslash\{0\}, \mathfrak{r}^{\infty} \in \mathbb{R}_{>}^{n}$ and $\Phi^{\infty} \in \mathbf{C}^{0}\left(\mathbb{R}^{n}, \mathbb{R}^{l}\right)$ such that for each $\lambda^{\infty}>0$ and compact set $\mathfrak{C} \subset \mathbb{R}^{n} \backslash\{0\}$ there exists $\varepsilon^{\infty}>0$ for which

$$
\max _{w \in \mathfrak{C}}\left\|\varepsilon^{-\mathfrak{e}^{\infty}} \diamond \phi\left(\varepsilon^{\mathfrak{r}^{\infty}} \diamond w\right)-\Phi^{\infty}(w)\right\| \leqslant \lambda^{\infty}
$$

for all $\varepsilon \geqslant \varepsilon^{\infty}$.

The function

$$
\phi(x):=\left(\begin{array}{c}
x_{2} \\
x_{1}^{2}+x_{2}^{3}+x_{2}^{\frac{1}{2}}
\end{array}\right)
$$

is homogeneous in the $\infty$-limit with triple

$$
\left(\mathfrak{r}^{\infty}, \mathfrak{e}^{\infty}, \Phi^{\infty}\right)=\left(\left(\begin{array}{c}
1 \\
\frac{2}{3}
\end{array}\right),\left(\begin{array}{l}
\frac{2}{3} \\
2
\end{array}\right),\left(\begin{array}{c}
x_{2} \\
x_{1}^{2}+x_{2}^{3}
\end{array}\right)\right)
$$

Note that $\phi$ is not homogeneous in the $\infty$-limit (in the classical sense).

Remark 3.3: The approximating function $\Phi^{\infty}$ is homogeneous (in the generalized sense) with triple $\left(\mathfrak{r}^{\infty}, \mathfrak{e}^{\infty}, \Phi^{\infty}\right)$. Indeed, for each $\mu>0$ and $w \in \mathbb{R}^{n}$

$$
\frac{\Phi_{i}^{\infty}\left(\mu^{\mathfrak{r}^{\infty}} \diamond w\right)}{\mu^{\mathfrak{e}_{i}^{\infty}}}=\lim _{\varepsilon \rightarrow+\infty} \frac{\phi_{i}^{\infty}\left((\varepsilon \mu)^{\mathfrak{r}^{\infty}} \diamond w\right)}{(\varepsilon \mu)^{\mathfrak{e}_{i}^{\infty}}}=\Phi_{i}^{\infty}(w(5)
$$

口

Definition 3.3: (Generalized homogeneity in the 0-limit). A function $\phi \in \mathbf{C}^{0}\left(\mathbb{R}^{n}, \mathbb{R}^{l}\right)$ is said to be homogeneous (in the generalized sense) in the 0-limit with triple $\left(\mathfrak{r}^{0}, \mathfrak{e}^{0}, \Phi^{\infty}\right)$ (weights $\mathfrak{r}^{0}$, degrees $\mathfrak{e}^{0}$ and approximating functions $\Phi^{0}$ ) if there exist $\mathfrak{e}^{0} \in \mathbb{R}_{\geqslant}^{l} \backslash\{0\}, \mathfrak{r}^{0} \in \mathbb{R}_{>}^{n}$ and $\Phi^{0} \in \mathbf{C}^{0}\left(\mathbb{R}^{n}, \mathbb{R}^{l}\right)$ such that for each $\lambda^{0}>0$ and compact set $\mathfrak{C} \subset \mathbb{R}^{n} \backslash\{0\}$ there exists $\varepsilon^{0}>0$ for which

$$
\max _{w \in \mathfrak{C}}\left\|\varepsilon^{-\mathfrak{e}^{0}} \diamond \phi\left(\varepsilon^{\mathfrak{r}^{0}} \diamond w\right)-\Phi^{0}(w)\right\| \leqslant \lambda^{0}
$$

for all $\varepsilon \leqslant \varepsilon^{0}$.
The function

$$
\phi(x):=\left(\begin{array}{c}
x_{2} \\
x_{1}^{2}+x_{2}^{3}+x_{2}^{\frac{1}{2}}
\end{array}\right)
$$

is homogeneous (in the generalized sense) in the 0-limit with triple

$$
\left(\mathfrak{r}^{0}, \mathfrak{e}^{0}, \Phi^{0}\right)=\left(\left(\begin{array}{l}
1 \\
4
\end{array}\right),\left(\begin{array}{l}
4 \\
2
\end{array}\right),\left(\begin{array}{c}
x_{2} \\
x_{1}^{2}+x_{2}^{\frac{1}{2}}
\end{array}\right)\right)
$$

Note that $\phi$ is not homogeneous in the 0-limit (in the classical sense).

Definition 3.4: (Generalized homogeneity in the bi-limit). A function $\phi \in \mathbf{C}^{0}\left(\mathbb{R}^{n}, \mathbb{R}^{l}\right)$ is said to be homogeneous (in the generalized sense) in the bi-limit with triple $\left(\mathfrak{r}^{\infty}, \mathfrak{e}^{\infty}, \Phi^{\infty}\right)$ and $\left(\mathfrak{r}^{0}, \mathfrak{e}^{0}, \Phi^{0}\right)$ if it is homogeneous (in the generalized sense) in the $\infty$-limit with triple $\left(\mathfrak{r}^{\infty}, \mathfrak{e}^{\infty}, \Phi^{\infty}\right)$ and homogeneous (in the generalized sense) in the 0-limit with triple $\left(\mathfrak{r}^{0}, \mathfrak{e}^{0}, \Phi^{0}\right)$.

Remark 3.4: Definitions 3.2, 3.3 and 3.4 generalize the definition of homogeneity in the $\infty$-limit, 0-limit and bilimit (in the classical sense: [2]). Weigths, degrees and approximating functions are not uniquely defined. $\square$

Consider the vector field $\phi(x):=\left(x_{2}^{\mathfrak{p}}, \mathfrak{c}^{0} x_{2}^{\mathfrak{q}^{0}}+\mathfrak{c}^{\infty} x_{2}^{\mathfrak{q}^{\infty}}\right)$ with $\mathfrak{p}>0,0<\mathfrak{q}^{0}<\mathfrak{q}^{\infty}$ and $\mathfrak{c}^{\infty}, \mathfrak{c}^{0} \geqslant 0$. It can be seen that $\phi$ is homogeneous (in the generalized sense) in the bi-limit with triple $\left(\left(1, \frac{2}{\mathfrak{p}}\right),\left(1, \max \left\{1, \frac{2\left(\mathfrak{q}^{p}-1\right)}{\mathfrak{p}}\right),\left(x_{2}^{\mathfrak{p}}, \mathfrak{c}^{p} x_{2}^{\mathfrak{q}^{p}}\right)\right.\right.$, $p=0, \infty$. In comparison with the notion of homogeneity in the bi-limit of [2] it is not required that $\mathfrak{q}^{0}, \mathfrak{q}^{\infty}<2$.

Remark 3.5: (Triangular maps are homogeneous in the bi-limit). Homogeneity (in the generalized sense) in the bilimit significantly enlarges the class of homogeneous maps (in the classical sense) in the bi-limit. In particular, triangular maps $\phi$ are always homogeneous (in the generalized sense) in the bi-limit with some triple $\left(\mathfrak{r}^{p}, \mathfrak{e}^{p}, \Phi^{p}\right), p=0, \infty$. Similar facts hold for $\phi$ norm-bounded by triangular maps. $\square$

From now on we will omit the term "in the generalized sense".

\section{HOMOGENEOUS STATE FEEDBACKS FOR A CHAIN OF POWER INTEGRATORS}

The notion of homogeneity (in the generalized sense) in the bi-limit is instrumental to introduce new stabilization methods. Throughout this section we consider a chain of $n$ power integrators, i.e.

$$
\dot{x}=A x^{\mathfrak{p}}+B u
$$

where $A \in \mathbb{R}^{n \times n}, B \in \mathbb{R}^{n \times 1}, \mathfrak{p} \in \mathbb{R}_{>}^{n}$ such that $\mathfrak{p}_{j}$, $j=1, \ldots, n$, is the ratio of odd numbers and $(A, B)$ is in Brunowskii form. For any non-decreasing sequence of reals $\left\{\mathfrak{d}_{j}^{\infty}\right\}_{j=1, \ldots, n}$ (resp. non-increasing sequence of reals $\left\{\mathfrak{d}_{j}^{0}\right\}_{j=1, \ldots, n}$ ) we see that to obtain homogeneity (in the generalized sense) of the vector field associated to (8) with degrees $\left(\mathfrak{r}^{\infty}+\mathfrak{d}^{\infty}\right)$ (resp. $\left.\left(\mathfrak{r}^{0}+\mathfrak{d}^{0}\right)\right)$ and weights $\mathfrak{r}^{\infty}$ (resp. $\mathfrak{r}^{0}$ ) we must choose $\mathfrak{r}^{\infty}$ (resp. $\mathfrak{r}^{0}$ ) as follows

$$
\mathfrak{r}_{j}^{p}=\frac{\mathfrak{r}_{j-1}^{p}+\mathfrak{d}_{j-1}^{p}}{\mathfrak{p}_{j}}, j=2, \ldots, n, p=0, \infty .
$$


Also, in this context it is natural to require that

$$
\mathfrak{r}_{i}^{p}+\mathfrak{d}_{i}^{p} \geqslant 0, \forall i=1, \ldots, n, p=0, \infty .
$$

$\left(\mathfrak{r}_{i}^{p}+\mathfrak{d}_{i}^{p}\right.$ is the degree of the $i$-th component of the vector field associated to (8)).

It is known that (8) can be rendered homogeneous (in the classical sense) in the bi-limit by using a stabilizing homogeneous in the bi-limit state feedback which can be designed by backstepping ([2]) and a converse Lyapunov result for homogeneous systems ([11]). In this section we show that this property can be extended to the case of homogeneity (in the generalized sense) in the bi-limit. More precisely, we show that there exists a homogeneous (in the generalized sense) in the bi-limit function $\alpha(x)$ such that the vector field $f:=A x+B \alpha$ (associated to the closedloop system (8) with $u=\alpha$ ) is homogeneous (in the generalized sense) in the bi-limit with triple $\left(\mathfrak{r}^{p}, \mathfrak{r}^{p}+\mathfrak{d}^{p}, \mathfrak{F}^{p}\right)$, $p=0, \infty$. We will do this by first defining a homogeneous in the bi-limit control law for (8) and then a homogeneous in the bi-limit Lyapunov function $V$ for the closed-loop system. We do not follow the step-by-step approach of [2], which requires at each step a converse Lyapunov result for homogeneous systems ([11]), and give the controller in one step. This provides new types of controllers even in the case of homogeneity in the bi-limit in the classical sense.

\section{A. Definition of the state feedback law}

Let $\mathfrak{X}^{j}:=\left(x_{1}, \ldots, x_{j}\right)$ and $\mathfrak{p}_{1}:=1$. Define recursively the following function

$$
\begin{aligned}
& \alpha(x):=\alpha^{n}\left(\mathfrak{X}^{n}\right) \\
& \alpha^{j}\left(\mathfrak{X}^{j}\right):=\left[e^{-\left(\zeta^{j, 0}\right)^{\mathfrak{a}\left(\mathbf{r}_{j}^{0}+\mathfrak{o}_{j}^{0}+\mathfrak{p}_{i} \mathbf{r}_{j}^{0}\right)}\left(\zeta^{j, \infty}\right)^{\mathfrak{m}_{j}}}\left(\alpha^{j, 0}\left(\mathfrak{X}^{j}\right)\right)^{\mathfrak{a}}\right. \\
& \left.+\left(1-e^{-\left(\zeta^{j, 0}\right)^{\mathfrak{a}\left(\mathbf{r}_{j}^{0}+\mathfrak{o}_{j}^{0}+\mathfrak{p}_{j} \mathbf{r}_{j}^{0}\right)}\left(\zeta^{j, \infty}\right)^{\mathfrak{m}_{j}}}\right)\left(\alpha^{j, \infty}\left(\mathfrak{X}^{j}\right)\right)^{\mathfrak{a}}\right]^{\frac{1}{\mathfrak{a}}} \\
& j=1, \ldots, n-1, \alpha^{0}:=0,
\end{aligned}
$$

with

$$
\begin{aligned}
& \alpha^{j, p}\left(\mathfrak{X}^{j}\right):=-\gamma_{j}\left(\zeta^{j, p}\left(\mathfrak{X}^{j}\right)\right)^{\mathfrak{r}_{j}^{p}+\mathfrak{o}_{j}^{p}-\mathfrak{p}_{j} \mathfrak{r}_{j}^{p}} . \\
& \cdot\left(x_{j}^{\mathfrak{a} \mathfrak{p}_{j}}-\left(\alpha^{j-1}\right)^{\mathfrak{a}}\left(\mathfrak{X}^{j-1}\right)\right)^{\frac{1}{\mathfrak{a}}}, \\
& \zeta^{j, p}\left(\mathfrak{X}^{j}\right)=:\left(\sum_{i=1}^{j}\left|x_{i}^{\mathfrak{a} \mathfrak{p}_{i}}-\left(\alpha^{i-1}\right)^{\mathfrak{a}}\left(\mathfrak{X}^{i-1}\right)\right|^{\frac{\mathfrak{b}}{\mathfrak{a} \mathfrak{p}_{i} \mathfrak{r}_{i}^{p}}}\right)^{\frac{1}{\mathfrak{b}}}
\end{aligned}
$$

for $p=0, \infty$ and $\mathfrak{a}, \mathfrak{b}, \mathfrak{m}_{i} \geqslant 1, i=1, \ldots, n$, such that

$$
\begin{aligned}
\mathfrak{a} & >\max _{\substack{k=1, \ldots, j \\
j=1, \ldots, n}} \frac{\mathfrak{r}_{k}^{0}}{\mathfrak{p}_{j} \mathfrak{r}_{j}^{0}} \\
\mathfrak{b} & >\max _{l=1, \ldots, n} \mathfrak{a p p}_{l} \mathfrak{r}_{l}^{p} \\
\mathfrak{m}_{i} & >\mathfrak{a} \max _{l=1, \ldots, i} \mathfrak{p}_{l} \mathfrak{r}_{l}^{\infty} \\
\mathfrak{m}_{i} & >\mathfrak{a}\left(\mathfrak{r}_{i}^{\infty}+\mathfrak{d}_{i}^{\infty}\right), i=1, \ldots, n,
\end{aligned}
$$

and $\gamma_{1}, \ldots, \gamma_{n} \geqslant 1$ are positive reals to be specified later.

Proposition 4.1: Let $\left\{\mathfrak{d}_{j}^{\infty}\right\}_{j=1, \ldots, n}$ (resp. $\left\{\mathfrak{d}_{j}^{0}\right\}_{j=1, \ldots, n}$ ) be non-decreasing (resp. non-increasing) sequence of reals and $\mathfrak{r}^{p}, p=0, \infty$, be as in (9) and satisfy (10). The vector field $f^{n}:=A x^{\mathfrak{p}}+B \alpha^{n}$ is continuous over $\mathbb{R}^{n}$ and homogeneous (in the generalized sense) in the bi-limit with triple $\left(\mathfrak{r}^{p}, \mathfrak{r}^{p}+\right.$ $\left.\mathfrak{d}^{p}, \mathfrak{F}^{n, p}\right), p=0, \infty$, where

$$
\begin{aligned}
& \mathfrak{F}_{i}^{n, p}:=x_{i+1}^{\mathfrak{p}_{i+1}}, i=1, \ldots, n-1, \\
& \mathfrak{F}_{n}^{j, p}:=-\gamma_{j}\left(\mathfrak{Z}^{j, p}\right)^{\mathfrak{a}\left(\mathfrak{r}_{j}^{p}+\mathfrak{d}_{j}^{p}-\mathfrak{p}_{j} \mathbf{r}_{j}^{p}\right)} . \\
& \cdot\left(x_{j}^{\mathfrak{a} \mathfrak{p}_{j}}-\left(\mathfrak{F}_{n}^{j-1, p}\right)^{\mathfrak{a}}\left(\mathfrak{X}^{j-1}\right)\right)^{\frac{1}{\mathfrak{a}}}, j=1, \ldots, n, \mathfrak{F}_{n}^{0, p}:=0,
\end{aligned}
$$

with

$$
\mathfrak{Z}^{j, p}=:\left(\sum_{i=1}^{j}\left|x_{i}^{\mathfrak{a} \mathfrak{p}_{i}}-\left(\mathfrak{F}_{n}^{i-1, p}\right)^{\mathfrak{a}}\right|^{\frac{\mathfrak{b}}{\mathfrak{a} \mathfrak{p}_{i} \mathbf{r}_{i}^{p}}}\right)^{\frac{1}{\mathfrak{b}}}
$$

Proof: We claim that $f^{n}$ is continuous over $\mathbb{R}^{n}$. It is sufficient to prove continuity of $\alpha^{n}$. Let do this by induction. Assume that $\alpha^{j-1}$ for some $j=2, \ldots, n$ is continuous over $\mathbb{R}^{j-1}$. By the induction hypothesis, on account of (10) and continuity of $\zeta^{j, p}, p=0, \infty$, over $\mathbb{R}^{j}$, since $\left|\left(\alpha^{j, p}\right)^{\mathfrak{a}}\right| \leqslant \gamma_{j}\left(\zeta^{j, p}\right)^{\mathfrak{a}\left(\mathfrak{r}_{j}^{p}+\mathfrak{o}_{j}^{p}\right)}$ for $p=0, \infty$ and for all $\mathfrak{X}^{j} \in \mathbb{R}^{j} \backslash\{0\}, \alpha^{j}$ is continuous over $\mathbb{R}^{j}$ with $\alpha^{j}(0)=0$. With similar arguments we prove continuity of $\alpha^{1}$ over $\mathbb{R}$. This concludes by induction the proof that $\alpha^{n}$ (and therefore $f^{n}$ ) is continuous over $\mathbb{R}^{n}$.

Let $\mathfrak{R}^{p}:=\left(\mathfrak{r}_{1}^{p}, \ldots, \mathfrak{r}_{j}^{p}\right)$. To prove the second part of the proposition, first we show by induction that $\alpha^{n}$ is homogeneous in the bi-limit with triple $\left(\mathfrak{r}^{p}, \mathfrak{r}_{n}^{p}+\mathfrak{d}_{n}^{p}, \mathfrak{F}_{n}^{n, p}\right)$, $p=0, \infty$.

Induction Step $(j-1) \cdot \alpha^{j-1}$ for some $j<n$ is homogeneous in the bi-limit with triple $\left(\mathfrak{R}^{j-1, p}, \mathfrak{r}_{j-1}^{p}+\right.$ $\left.\mathfrak{d}_{j-1}^{p}, \mathfrak{F}_{n}^{j-1, p}\right), p=0, \infty$.

We show that the induction step holds for $j$. This follows from the induction step, (10), propositions 1.1, 1.5, 1.6 (if $\mathfrak{r}_{j}^{p}+\mathfrak{d}_{j}^{p}-\mathfrak{p}_{j} \mathfrak{r}_{j}^{p} \geqslant 0$ ), $1.7\left(\right.$ if $\left.\mathfrak{r}_{j}^{p}+\mathfrak{d}_{j}^{p}-\mathfrak{p}_{j} \mathfrak{r}_{j}^{p}<0\right)$ and 1.8 and the fact that $\zeta^{j, p}$ is positive definite and radially unbounded over $\mathbb{R}^{j}$. In a similar way, we prove that $\alpha^{1}$ is homogeneous in the bi-limit with triple $\left(\mathfrak{r}_{1}^{p}, \mathfrak{r}_{1}^{p}+\mathfrak{d}_{1}^{p}, \mathfrak{F}_{n}^{1, p}\right), p=0, \infty$. Therefore, by induction we conclude that $\alpha^{n}$ is homogeneous in the bi-limit with triple $\left(\mathfrak{r}^{p}, \mathfrak{r}_{n}^{p}+\mathfrak{d}_{n}^{p}, \mathfrak{F}_{n}^{n, p}\right), p=0, \infty$. Using the definition (9) finally we establish that $f^{n}$ is homogeneous in the bi-limit with triple $\left(\mathfrak{r}^{p}, \mathfrak{r}^{p}+\mathfrak{d}^{p}, \mathfrak{F}^{n, p}\right), p=0, \infty$.

Remark 4.1: Notice that if $\mathfrak{r}_{k}^{0}<\mathfrak{r}_{j}^{0} \mathfrak{p}_{j}$ for all $j=1, \ldots, n$ and $k=1 \ldots, j$ and $p=0, \infty$ then a simple choice of $\mathfrak{a}$ is $\mathfrak{a}=1$. Also notice that when $\mathfrak{d}_{j}^{\infty}>\mathfrak{d}_{j}^{0}$ for all $j=1, \ldots, n$ on account of proposition 1.2 , the definition of $\alpha^{j}$ can be remarkably simplified as $\alpha^{j}:=\left[\left(\alpha^{j, 0}\right)^{\mathfrak{a}}+\left(\alpha^{j, \infty}\right)^{\mathfrak{a}}\right]^{\frac{1}{\mathfrak{a}}}$.

\section{B. Construction of the Lyapunov function}

Let

$$
(\mathfrak{d}, \mathfrak{h}) \in \mathbb{R}^{2} \mapsto \delta_{\mathfrak{d}, \mathfrak{h}}= \begin{cases}1 & \text { if } \mathfrak{d}=\mathfrak{h} \\ 0 & \text { otherwise }\end{cases}
$$

Define the following numbers

$$
\mathfrak{c}_{j}^{p}:=\mathfrak{w}^{p}-\mathfrak{d}_{j}^{p}-\mathfrak{r}_{j}^{p}-\mathfrak{a p}_{j} \mathfrak{r}_{j}^{p},
$$

$j=1, \ldots, n$, for some $\mathfrak{w}^{0}, \mathfrak{w}^{\infty}>0$ such that $\mathfrak{c}^{0}, \mathfrak{c}^{\infty}>0$ and

$$
\mathfrak{c}_{j}^{\infty}+\mathfrak{r}_{j}^{\infty}-\mathfrak{a} \mathfrak{p}_{j} \mathfrak{r}_{j}^{\infty}-\mathfrak{r}_{k}^{\infty}>\mathfrak{c}_{j}^{0}+\mathfrak{r}_{j}^{0}-\mathfrak{a p}_{j} \mathfrak{r}_{j}^{0}-\mathfrak{r}_{k}^{0}>0,
$$


$1 \leqslant k \leqslant j$, and recursively the following function

$$
\begin{aligned}
& V(x):=V^{n}\left(\mathfrak{X}^{n}\right), V^{j}\left(\mathfrak{X}^{j}\right):=V^{j-1}\left(\mathfrak{X}^{j-1}\right)+V^{j, 0}(x) \\
& +V^{j, \infty}(x), j=1, \ldots, n-1, V^{0}\left(x_{1}\right):=0,
\end{aligned}
$$

where

$$
\begin{aligned}
& V^{j, p}\left(\mathfrak{X}^{j}\right):=\left(\zeta^{j, p}\right)^{\mathfrak{c}_{j}^{p}}\left(\mathfrak{X}^{j}\right) \cdot \\
& \cdot \int_{\left(\alpha^{j-1}\left(\mathfrak{X}^{j-1}\right)\right)^{\frac{1}{\mathfrak{p}_{j}}}\left[s^{\mathfrak{a} \mathfrak{p}_{j}}-\left(\alpha^{j-1}\left(\mathfrak{X}^{j-1}\right)\right)^{\mathfrak{a}}\right] d s, p=0, \infty .}^{x_{j}} .
\end{aligned}
$$

We have the following result.

Proposition 4.2: Let $\left\{\mathfrak{d}_{j}^{\infty}\right\}_{j=1, \ldots, n}$ (resp. $\left\{\mathfrak{d}_{j}^{0}\right\}_{j=1, \ldots, n}$ ) be non-decreasing (resp. non-increasing) sequence of reals and $\mathfrak{r}^{p}, p=0, \infty$, be as in (9) and satisfy (10). Moreover, let $\mathfrak{m}$, $\mathfrak{a}$ and $\mathfrak{b}$ satisfy (14), (13) and (15) and $\mathfrak{c}^{p}, p=0, \infty$, be as in (19) and satisfy (20). The function $V^{n}$ is positive definite and radially unbounded over $\mathbb{R}^{n}$ and $\left(\frac{\partial V^{n}}{\partial x}\right)^{T}$ is continuous over $\mathbb{R}^{n}$ and homogeneous (in the generalized sense) in the bilimit with triple $\left(\mathfrak{r}^{p},\left(\mathfrak{w}^{p}-\mathfrak{d}_{1}^{p}-\mathfrak{r}_{1}^{p}, \ldots, \mathfrak{w}^{p}-\mathfrak{d}_{n}^{p}-\mathfrak{r}_{n}^{p}\right), \mathfrak{V}^{n, p}\right)$, $p=0, \infty$.

\section{Proof:}

We proceed by induction. As usual let $\mathfrak{R}^{p}:=$ $\left(\mathfrak{r}_{1}^{p}, \ldots, \mathfrak{r}_{j}^{p}\right)^{T}, p=0, \infty$.

Induction Step $(j-1)$. For some integer $j \in[2, n]$ the function $V^{j-1}$ is positive definite and radially unbounded over $\mathbb{R}^{j-1}$, $\left(\frac{\partial V^{j-1}}{\partial \mathfrak{X}^{j-1}}\right)^{T}$ is continuous over $\mathbb{R}^{j-1}$, with $\left(\frac{\partial V^{j-1}}{\partial \mathfrak{X}^{j}-1}\right)^{T}(0)=0$, and homogeneous (in the generalized sense) in the bi-limit with triple $\left(\mathfrak{R}^{j-1, p},\left(\mathfrak{w}^{p}-\right.\right.$ $\left.\left.\mathfrak{d}_{1}^{p}-\mathfrak{r}_{1}^{p}, \ldots, \mathfrak{w}^{p}-\mathfrak{d}_{j-1}^{p}-\mathfrak{r}_{j-1}^{p}\right),\left(\mathfrak{V}_{1}^{n, p}, \ldots, \mathfrak{V}_{j-1}^{n, p}\right)^{T}\right), p=$ $0, \infty$, and $\left(\frac{\partial\left(\alpha^{i}\right)^{\mathfrak{a}}}{\partial \mathfrak{X}^{i}}\right)^{T}, i=1, \ldots, j-1$, is continuous over $\mathbb{R}^{i}$, with $\left(\frac{\partial\left(\alpha^{i}\right)^{\mathfrak{a}}}{\partial \mathfrak{X}^{i}}\right)^{T}(0)=0$, and homogeneous in the bi-limit with triple $\left(\mathfrak{R}^{i, p}, \mathfrak{a} \mathfrak{p}_{i+1} \mathfrak{r}_{i+1}^{p}-\mathfrak{r}_{1}^{p}, \ldots, \mathfrak{a} \mathfrak{p}_{i+1} \mathfrak{r}_{i+1}^{p}-\right.$ $\left.\mathfrak{r}_{i}^{p},\left(\mathfrak{D}_{1}^{i, p}, \ldots, \mathfrak{D}_{i}^{i, p}\right)^{T}\right), p=0, \infty$.

We show that the induction step holds for $j$.

( $V^{j}$ is proper and radially unbounded over $\mathbb{R}^{j}$ ). Since $\zeta^{j, p}$, $p=0, \infty$, is positive definite and radially unbounded over $\mathbb{R}^{j}$ and by the induction hypothesis $\left(V^{j-1}\right.$ is positive definite and radially unbounded over $\mathbb{R}^{j-1}$ ), $V^{j}$ is positive definite and radially unbounded over $\mathbb{R}^{j}$.

$\left(\left(\frac{\partial V^{j}}{\partial \mathfrak{X}^{j}}\right)^{T}\right.$ is continuous over $\left.\mathbb{R}^{j}\right)$. Use the induction hypothesis, (9), (10), (13), (14), (15), (20) and the fact that $\zeta^{i, p}$, $1 \leqslant i \leqslant j$ and $p=0, \infty$, is positive and continuous over $\mathbb{R}^{i} \backslash\{0\}$ and $\zeta^{l, p} \leqslant \zeta^{j, p}, p=0, \infty$, for all $l \leqslant i \leqslant j-1$ and $\mathfrak{X}^{j} \in \mathbb{R}^{j}$.

( Homogeneity of $\left(\frac{\partial V^{j}}{\partial \mathfrak{X}^{j}}\right)^{T}$ ). Use the induction hypothesis, (16), (20) and propositions 1.1, 1.2, 1.4, 1.5 and 1.8.

This proves the induction step for $j$. It is easy to see that $V^{1}$ is positive definite and radially unbounded over $\mathbb{R}, \frac{\partial V^{1}}{\partial x_{1}}$ is continuous over $\mathbb{R}$ and homogeneous (in the generalized sense) in the bi-limit with triple $\left(\mathfrak{r}_{1}^{p}, \mathfrak{w}^{p}-\mathfrak{d}_{1}^{p}-\mathfrak{r}_{1}^{p}, \mathfrak{V}_{1}^{n, p}\right)$, $p=0, \infty$. This concludes the proof by induction.

As a second step, we prove that the derivative of $V$ along the trajectories of the closed-loop system (8) with $u=\alpha^{n}$ is negative definite.

Proposition 4.3: Let $\left\{\mathfrak{d}_{j}^{\infty}\right\}_{j=1, \ldots, n}$ (resp. $\left\{\mathfrak{d}_{j}^{0}\right\}_{j=1, \ldots, n}$ ) be non-decreasing (resp. non-increasing) sequence of reals and $\mathfrak{r}^{p}, p=0, \infty$, be as in (9) and satisfy (10). Moreover, let $\mathfrak{m}, \mathfrak{a}$ and $\mathfrak{b}$ satisfy (14), (13) and (15) and $\mathfrak{c}^{p}, p=0, \infty$, be as in (19) and satisfy (20). The function $\left(\frac{\partial V^{n}}{\partial \mathfrak{X}^{n}}\right) f^{n}$ is homogeneous (in the generalized sense) in the bi-limit with triple $\left(\mathfrak{r}^{p}, \mathfrak{w}^{p},\left(\frac{\partial V^{n}}{\partial x} f^{n}\right)^{p}\right), p=0, \infty$, where $\left(\frac{\partial V^{n}}{\partial x} f^{n}\right)^{p}:=$ $\sum_{i=1}^{n} \mathfrak{V}_{i}^{n, p} \mathfrak{F}_{i}^{n, p}$. Moreover, there exist $\gamma_{1}^{*}, \ldots, \gamma_{n}^{*}>0$ such that

$$
\left(\frac{\partial V^{n}}{\partial x} f^{n}\right)(x)<0,\left(\frac{\partial V^{n}}{\partial x} f^{n}\right)^{p}(x)<0, \quad p=0, \infty,
$$

for all $x \neq 0$ and for all $\gamma_{j} \geqslant \gamma_{j}^{*}, j=1, \ldots, n$.

Proof: We proceed by induction. Let $f^{j}\left(\mathfrak{X}^{j}\right):=$ $\left(x_{2}^{\mathfrak{p}_{2}}, \cdots, x_{j}^{\mathfrak{p}_{j}}, \alpha^{j}\left(\mathfrak{X}^{j}\right)\right)^{T}$ with $f^{1}\left(x_{1}\right):=\alpha_{1}\left(x_{1}\right)$. Moreover, $\mathfrak{R}^{j, p}:=\left(\mathfrak{r}_{1}^{p}, \ldots, \mathfrak{r}_{j}^{p}\right)^{T}, p=0, \infty$.

Induction Hypothesis. $\left(\frac{\partial V^{j}}{\partial \mathfrak{X}^{j}}\right) f^{j}$ is for some $j \in[1, n-1]$ homogeneous in the bi-limit with triple $\left(\mathfrak{R}_{k}^{p}, \mathfrak{w}^{p},\left(\frac{\partial V^{j}}{\partial x} f^{j}\right)^{p}\right)$, $p=0, \infty$, where $\left(\frac{\partial V^{j}}{\partial x} f^{j}\right)^{p}:=\sum_{i=1}^{j} \mathfrak{V}_{i}^{n, p} \mathfrak{F}_{i}^{n, p}$. Moreover, there exist $\gamma_{1}^{*}, \ldots, \gamma_{j}^{*}>0$ such that

$$
\left(\frac{\partial V^{j}}{\partial \mathfrak{X}^{j}} f^{j}\right)\left(\mathfrak{X}^{j}\right)<0,\left(\frac{\partial V^{j}}{\partial \mathfrak{X}^{j}} f^{j}\right)^{p}\left(\mathfrak{X}^{j}\right)<0, \quad p=0, \infty
$$

for all $\mathfrak{X}^{j} \in \mathbb{R}^{j} \backslash\{0\}$ and for all $\gamma_{i} \geqslant \gamma_{i}^{*}, i=1, \ldots, j$.

We will prove the induction step for $j+1$. Notice that

$$
\begin{aligned}
& \frac{\partial V^{j+1}}{\partial \mathfrak{X}^{j+1}} f^{j+1}=\frac{\partial V^{j+1}}{\partial \mathfrak{X}^{j}}\left(\begin{array}{c}
x_{2}^{\mathfrak{p}_{2}} \\
\vdots \\
x_{j+1}^{\mathfrak{p}_{j+1}}
\end{array}\right)+\frac{\partial V^{j+1}}{\partial x_{j+1}} \alpha^{j+1} \\
& :=\xi^{j+1}-\gamma_{j+1} \sigma^{j+1}
\end{aligned}
$$

From proposition 4.2 and since $x_{j+1}-\left(\alpha^{j}\right)^{\frac{1}{\mathfrak{p}_{j+1}}}$ and $x_{j+1}^{\mathfrak{a} \mathfrak{p}_{j+1}}-$ $\left(\alpha^{j}\right)^{\mathfrak{a}}$ as well as $x_{j+1}-\left(\mathfrak{F}_{j}\right)^{\frac{1}{\mathfrak{p}_{j+1}}}$ and $x_{j+1}^{\mathfrak{a p}_{j+1}}-\left(\mathfrak{F}_{j}\right)^{\mathfrak{a}}$ have the same sign, it follows that $\sigma^{j+1}$ is nonnegative and it is zero only at points $\mathfrak{X}^{j} \in \mathbb{R}^{j} \backslash\{0\}: x_{j+1}-\alpha^{j}=0$, as well as $\mathfrak{w}^{j+1, p}, p=0, \infty$, is nonnegative and zero only at points $\mathfrak{X}^{j} \in \mathbb{R}^{j} \backslash\{0\}: x_{j+1}-\mathfrak{F}_{j+1}^{k, p}=0$. This together with the induction hypothesis implies that

$$
\begin{aligned}
& \left\{\mathfrak{X}^{j+1} \in \mathbb{R}^{j+1} \backslash\{0\}: \sigma^{j+1}=0\right\} \\
& \subset\left\{\mathfrak{X}^{j+1} \in \mathbb{R}^{j+1} \backslash\{0\}: \xi^{j+1}<0\right\} \\
& \left\{\mathfrak{X}^{j+1} \in \mathbb{R}^{j+1} \backslash\{0\}: \mathfrak{w}^{j+1, p}=0\right\} \\
& \subset\left\{\mathfrak{X}^{j+1} \in \mathbb{R}^{j+1} \backslash\{0\}: \Xi^{j+1, p}<0\right\}, \quad p=0, \infty,
\end{aligned}
$$

for all $\gamma_{i} \geqslant \gamma_{i}^{*}, i=1, \ldots, j$. By application of proposition 1.9 to $\xi^{j+1}$ and $\sigma^{j+1}$ it is concluded that there exist $\gamma_{1}^{*}, \ldots, \gamma_{j+1}^{*}>0$ such that

$$
\begin{aligned}
& \left(\frac{\partial V^{j+1}}{\partial \mathfrak{X}^{j+1}} f^{j+1}\right)<0 \\
& \left(\frac{\partial V^{j+1}}{\partial \mathfrak{X}^{j+1}} f^{j+1}\right)_{j}^{p}<0, \quad j=1, \ldots, j+1, \quad p=0, \infty
\end{aligned}
$$

for all $\mathfrak{X}^{j+1} \in \mathbb{R}^{j+1} \backslash\{0\}$ and for all $\gamma_{i} \geqslant \gamma_{i}^{*}, i=1, \ldots, j+$ 1 , i.e. the induction hypothesis for $j+1$.

We conclude the proof of the proposition by induction. It is easy to see that $\frac{\partial V^{1}}{\partial x_{1}} f^{1}$ is homogeneous (in the generalized 
sense) in the bi-limit with triple $\left(\mathfrak{r}^{p}, \mathfrak{w}^{p},\left(\frac{\partial V^{1}}{\partial x_{1}} f^{1}\right)^{p}\right), p=$ $0, \infty$, where $\left(\frac{\partial V^{1}}{\partial x^{1}} f^{1}\right)^{p}:=\mathfrak{V}_{1}^{1, p} \mathfrak{F}_{1}^{1, p}$. Moreover,

$$
\left(\frac{\partial V^{1}}{\partial x_{1}} f^{1}\right)<0,\left(\frac{\partial V^{1}}{\partial x_{1}} f^{1}\right)^{p}<0, p=0, \infty,
$$

for all $x_{1} \neq 0$ and for any $\gamma_{1}>0$. This completes the proof of the proposition by induction.

\section{RESULTS ON STATE FEEDBACK STABILIZATION AND ROBUSTNESS}

The following result is the key result for establishing the global asymptotic stability of a given system from the global asymptotic stability of its approximations at zero and at infinity.

Proposition 5.1: Assume

(i) the existence of positive definite and radially unbounded $V: \mathbb{R}^{n} \rightarrow \mathbb{R}$ such that $\left(\frac{\partial V}{\partial x}\right)^{T}$ is continuous over $\mathbb{R}^{n}$ and homogeneous (in the generalized sense) in the bi-limit with triple $\left(\mathfrak{r}^{p},\left(\mathfrak{w}^{p}-\mathfrak{d}_{1}^{p}-\right.\right.$ $\left.\left.\mathfrak{r}_{1}^{p}, \ldots, \mathfrak{w}^{p}-\mathfrak{d}_{n}^{p}-\mathfrak{r}_{n}^{p}\right), \mathfrak{V}^{p}\right), p=0, \infty$,

(ii) $f: \mathbb{R}^{n} \rightarrow \mathbb{R}$ and $g: \mathbb{R}^{n} \rightarrow \mathbb{R}$ are continuous and homogeneous (in the generalized sense) in the bi-limit with triple $\left(\mathfrak{r}^{p}, \mathfrak{d}^{f, p}+\mathfrak{r}^{p}, \mathfrak{F}^{p}\right), p=0, \infty$, and, respectively, $\left(\mathfrak{r}^{p}, \mathfrak{d}^{g, p}+\mathfrak{r}^{p}, \mathfrak{G}^{p}\right), p=0, \infty$, with $\mathfrak{d}_{j}^{f, \infty} \geqslant \mathfrak{d}_{j}^{g, \infty}, \mathfrak{d}_{j}^{f, 0} \leqslant \mathfrak{d}_{j}^{g, 0}, j=1, \ldots, n$,

(iii) for all nonzero $x$

$$
\left(\frac{\partial V}{\partial x} f\right)(x)<0,\left(\frac{\partial V}{\partial x} f\right)^{p}(x)<0, \quad p=0, \infty
$$

where $\left(\frac{\partial V}{\partial x} f\right)^{0}$ and $\left(\frac{\partial V}{\partial x} f\right)^{\infty}$ are the approximating functions associated with $\frac{\partial V}{\partial x} f$ at zero and, respectively, at infinity.

There exists $c^{*}>0$ such that the origin of $\dot{x}=f(x)+c g(x)$ is globally asymptotically stable for all $c \in\left[0, c^{*}\right]$.

From this proposition, together with proposition 4.2 and 1.2 , it readily follows a stabilization result on a chain of power integrators with a nonlinear additive perturbation.

Theorem 5.1: Let $\left\{\mathfrak{d}_{j}^{\infty}\right\}_{j=1, \ldots, n}$ (resp. $\left\{\mathfrak{d}_{j}^{0}\right\}_{j=1, \ldots, n}$ ) be non-decreasing (resp. non-increasing) sequence of reals and $\mathfrak{r}^{p}, p=0, \infty$, be as in (9) and satisfy (10) and consider the system $\dot{x}=A x^{\mathfrak{p}}+B u$, where $A \in \mathbb{R}^{n \times n}, B \in \mathbb{R}^{n \times 1}$ and $(A, B)$ is in Brunowskii form. Assume

(i) $g: \mathbb{R}^{n} \rightarrow \mathbb{R}$ is continuous and homogeneous (in the generalized sense) in the bi-limit with triple $\left(\mathfrak{r}^{p}, \mathfrak{v}^{p}+\mathfrak{r}^{p}, \mathfrak{G}^{p}\right), p=0, \infty$, with $\mathfrak{d}_{j}^{\infty} \geqslant \mathfrak{v}_{j}^{\infty}, \mathfrak{d}_{j}^{0} \leqslant$ $\mathfrak{v}_{j}^{0}, j=1, \ldots, n$.

There exist $c^{*}, \gamma_{1}^{*}, \ldots, \gamma_{n}^{*}>0$ such that the origin of $\dot{x}=$ $A x^{\mathfrak{p}}+B u+c g(x)$ with $u=\alpha(x)$ and $\alpha$ defined in (11), is globally asymptotically stable for all $c \in\left[0, c^{*}\right]$ and $\gamma_{j} \geqslant$ $\gamma_{j}^{*}, j=1, \ldots, n$.

\section{CONCLUSIONS}

In this paper we have introduced a generalized notion of homogeneity and homogeneous approximations. Generalized homogeneity has most of the properties of classical homogeneity but captures a much larger class of nonlinear maps. Generalized homogeneity allows to design homogeneous state feedback stabilizers for vector fields such as chain of integrators and then stabilization is retained despite of nonlinearities with a homogeneity degree greater at the origin and smaller at infinity of the one of the chain of integrators. Further study will be devoted to output feedback stabilization.

\section{REFERENCES}

[1] S. Battilotti, Incremental generalized homogeneity, observer design and semi global stabilization, Asian Journal of Control, publication on-line: April 2013.

[2] V. Andrieu, L. Praly, A. Astolfi, Homogeneous approximation recursive observer and output feedback, SIAM Journ. Contr. Optim., 47, 1814-1850, 2008.

[3] A. Bacciotti, L. Rosier, Lyapunov functions and stability in control theory, LNCS, 267, Springer, Berlin, 2001.

[4] W. Hahn, Stability of motion, Springer, Berlin, 1967.

[5] H. Hermes, Homogeneous coordinates and continuous asymptotically stabilizing feedback control, Diff. Equat. Stab. Contr., S. Elaydi Ed., LCAM, 109, Marcel Dekker, NY, 249-260, 1991.

[6] M. Kawski, Stabilization of nonlinear systems in the plane, Ann. Math., 12, 169-175, 1989.

[7] J.L Massera, Contributions to stability theory, Syst. Contr. Lett., 64, 182-206, 1956

[8] C. Qian, A homogeneous domination approach for global output feedback stabilization of a class of nonlinear systems, ACC, Portland, 2005

[9] C. Qian, W. Lin, Output feedback control of a class of nonlinear systems: a non separation principle paradigm, IEEE Trans. Autom. Contr., 47, 1710-1719, 2001.

[10] C. Qian, W. Lin, Observer design, homogeneous approximation and nonsmooth output feedback stabilization of nonlinear systems, IEEE Trans. Autom. Contr., 51, 1457-1471, 2006.

[11] L. Rosier, Homogeneous Lyapunov function for homogeneous continuous vector field, Syst. Contr. Lett., 19, 467-473, 1992.

[12] B. Yang, W. Lin, Homogeneous observers, iterative design and global stabilization of high-order a nonlinear systems by smooth output feedback, IEEE Trans. Autom. Contr., 49, 2004.

\section{APPENDIX}

Throughout we will omit the term "in the generalized sense".

1) Linear combinations of homogeneous functions: The following results can be proved as a direct consequence of our definitions.

Proposition 1.1: (Sum of functions). Assume that $\phi, \psi$ : $\mathbb{R}^{n} \rightarrow \mathbb{R}^{l}$ are homogeneous in the bi-limit with triple $\left(\mathfrak{r}^{\phi, p}, \mathfrak{v}^{\phi, p}, \Phi^{p}\right), p=0, \infty$, and, respectively, $\left(\mathfrak{r}^{\psi, p}, \mathfrak{v}^{\psi, p}, \Psi^{p}\right), p=0, \infty$ such that for all $i=1, \ldots, l$ and $j=1, \ldots, n$

$$
\frac{\mathfrak{v}_{i}^{\phi, p}}{\mathfrak{r}_{j}^{\phi, p}}=\frac{\mathfrak{v}_{i}^{\psi, p}}{\mathfrak{r}_{j}^{\psi, p}}
$$

The function $\phi+\psi$ is homogeneous in the bi-limit with triple $\left(\mathfrak{r}^{\phi, p}, \mathfrak{v}^{\phi, p}, \Phi^{p}+\Psi^{p}\right), p=0, \infty$.

Proposition 1.2: (Domination). Assume that $\phi, \psi$ : $\mathbb{R}^{n} \rightarrow \mathbb{R}^{l}$ are homogeneous in the bi-limit with triple $\left(\mathfrak{r}^{\phi, p}, \mathfrak{v}^{\phi, p}, \Phi^{p}\right), \quad p=0, \infty$, and, respectively, $\left(\mathfrak{r}^{\psi, p}, \mathfrak{v}^{\psi, p}, \Psi^{p}\right), p=0, \infty$ such that for all $i=1, \ldots, l$ and $j=1, \ldots, n$

$$
\begin{aligned}
& \frac{\mathfrak{r}_{1}^{\psi, p}}{\mathfrak{r}_{1}^{\phi, p}}=\ldots=\frac{\mathfrak{r}_{n}^{\psi, p}}{\mathfrak{r}_{n}^{\phi, p}} \\
& \mathfrak{v}_{i}^{\phi, \infty}>\mathfrak{v}_{i}^{\psi, \infty}\left(\operatorname{resp} \cdot \mathfrak{v}_{i}^{\phi, 0}<\mathfrak{v}_{i}^{\psi, 0}\right)
\end{aligned}
$$


The function $\phi+\psi$ is homogeneous in the $\infty$-(resp. 0-)limit with triple $\left(\mathfrak{r}^{\infty}, \mathfrak{v}^{\phi, \infty}, \Phi^{\infty}\right)\left(\operatorname{resp} .\left(\mathfrak{r}^{0}, \mathfrak{v}^{\phi, 0}, \Phi^{0}\right)\right)$.

2) Absolute value: This proposition follows directly from the fact that ||$a|-| b|| \leqslant|a-b|$ for all $a, b \in \mathbb{R}$.

Proposition 1.3: (Absolute value of functions). Assume that $\phi: \mathbb{R}^{n} \rightarrow \mathbb{R}^{l}$ is homogeneous in the bi-limit with triple $\left(\mathfrak{r}^{\phi, p}, \mathfrak{v}^{\phi, p}, \Phi^{p}\right), p=0, \infty$. The function $\left(\left|\phi_{1}\right|, \ldots,\left|\phi_{n}\right|\right)^{T}$ is homogeneous in the bi-limit with triple $\left(\mathfrak{r}^{\Phi, p}, \mathfrak{v}^{\Phi, p},\left(\left|\Phi_{1}^{p}\right|, \ldots,\left|\Phi_{l}^{p}\right|\right)^{T}\right), p=0, \infty$.

3) Derivative and integral of homogeneous functions:

It is not possible to say anything on $\frac{\partial \phi}{\partial x_{i}}$ when dealing with homogeneity in the bi-limit. On the other hand, for the integral of a homogeneous $\phi: \mathbb{R}^{n} \rightarrow \mathbb{R}$ we have the following result, which is a direct consequence of the definition of homogeneity in the bi-limit.

Proposition 1.4: (Integral). Assume that $\phi$ : $\mathbb{R}^{n} \rightarrow \mathbb{R}$ is homogeneous in the bi-limit with triple $\left(\mathfrak{r}^{p}, \mathfrak{v}^{p}, \Phi^{p}\right), p=0, \infty$. The function $x \mapsto \psi(x):=$ $\int_{0}^{x_{l}} \phi\left(x_{1}, \ldots, x_{l-1}, s, x_{l+1}, \ldots, x_{n}\right) d s, l=1, \ldots, n$, is homogeneous in the bi-limit with triple $\left(\mathfrak{r}^{p}, \mathfrak{v}^{p}+\mathfrak{r}_{l}^{p}, \Psi^{p}\right)$ with $\Psi_{i}^{p}:=\int_{0}^{x_{l}} \Phi_{i}^{p}\left(x_{1}, \ldots, x_{l-1}, s, x_{l+1}, \ldots, x_{n}\right) d s$.

The following results can be proved as the analogue results in [2].

\section{4) Composition of homogeneous functions:}

Proposition 1.5: Assume that $\phi: \mathbb{R}^{n} \rightarrow \mathbb{R}$ is homogeneous in the bi-limit with triple $\left(\mathfrak{r}^{\phi, p}, \mathfrak{v}^{\phi, p}, \Phi^{p}\right), p=0, \infty$, with $\mathfrak{v}^{\phi, p}>0$ for all $j=1, \ldots, n$, and $Z: \mathbb{R} \rightarrow \mathbb{R}$ is homogeneous in the bi-limit with triple $\left(\mathfrak{r}^{\zeta, p}, \mathfrak{v}^{\zeta, p}, \mathfrak{Z}^{p}\right)$, $p=0, \infty$. The function $x \mapsto(Z \circ \phi)(x):=Z(\phi(x))$ is homogeneous in the bi-limit with triple $\left(\mathfrak{r}^{\phi, p}, \frac{\mathfrak{v}^{Z, p}}{\mathfrak{r}^{Z, p}} \mathfrak{v}^{\phi, p}, \Psi^{p}\right)$ with $\Psi_{i}^{p}:=\mathfrak{Z}^{p} \circ \Phi_{i}^{p}$.

5) Product and ratio of homogeneous functions:

Proposition 1.6: (Product). Assume that $\phi: \mathbb{R}^{n} \rightarrow$ $\mathbb{R}^{l}$ is homogeneous in the bi-limit with triple $\left(\mathfrak{r}^{p},\left(\mathfrak{v}^{p}-\right.\right.$ $\left.\left.\mathfrak{z}_{1}^{p}, \ldots, \mathfrak{v}^{p}-\mathfrak{z}_{n}^{p}\right), \Phi^{p}\right), p=0, \infty$, and $\psi: \mathbb{R}^{n} \rightarrow \mathbb{R}^{l}$ is homogeneous in the bi-limit with triple $\left(\mathfrak{r}^{p}, \mathfrak{z}^{p}, \Psi^{p}\right), p=$ $0, \infty$. The product $\phi^{T} \psi$ is homogeneous in the bi-limit with triple $\left(\mathfrak{r}^{p}, \mathfrak{v}^{p},\left(\phi^{T} \psi\right)^{p}\right), p=0, \infty$, where $\left(\phi^{T} \psi\right)^{p}:=$ $\sum_{h=1}^{n}\left(\Phi_{h}^{p} \Psi_{h}^{p}\right)$.

The following result follows from the fact that continuous functions are also uniformly continuous on compact subsets.

Proposition 1.7: (Ratio). Assume that

(I) $\beta: \mathbb{R}^{n} \rightarrow \mathbb{R}^{n}$ is homogeneous in the bi-limit with triple $\left(\mathfrak{r}^{p}, \mathfrak{v}^{p}, \mathfrak{B}^{p}\right), p=0, \infty$,

(II) $\alpha: \mathbb{R}^{n} \rightarrow \mathbb{R}^{n}, \phi: \mathbb{R}^{n} \rightarrow \mathbb{R}, x \mapsto \phi(x):=$ $\Phi(\alpha(x)+\beta(x))$, and $\psi: \mathbb{R}^{n} \rightarrow \mathbb{R}, x \mapsto \psi(x):=$ $\Psi(\alpha(x)+\beta(x))$ are such that

$$
\begin{aligned}
& \varepsilon^{-\mathfrak{e}^{\phi, p}} \phi\left(\varepsilon^{\mathfrak{r}^{p}} \diamond x\right)=\Phi^{p}\left(\mathfrak{A}^{p}(x)+\varepsilon^{-\mathfrak{v}^{p}} \diamond \beta\left(\varepsilon^{\mathfrak{r}^{p}} \diamond x\right)\right), \\
& \varepsilon^{-\mathfrak{e}^{\psi, p}} \psi\left(\varepsilon^{\mathfrak{r}^{p}} \diamond x\right)=\Psi^{p}\left(\mathfrak{A}^{p}(x)+\varepsilon^{-\mathfrak{v}^{p}} \diamond \beta\left(, \varepsilon^{\mathfrak{r}^{p}} \diamond x\right)\right)
\end{aligned}
$$

for some $\mathfrak{e}^{\phi, p}>\mathfrak{e}^{\psi, p}$ and for all $\varepsilon>0$ and $x \in$ $\mathbb{R}^{n} \backslash\{0\}$,

(III) $\frac{\Phi}{\psi}$ and $\frac{\Phi^{p}}{\Psi^{p}}:=\frac{\Phi\left(\mathfrak{A}^{p}(x)+\mathfrak{B}^{p}(x)\right)}{\Psi\left(\mathfrak{A}^{p}(x)+\mathfrak{B}^{p}(x)\right)}, p=0, \infty$, are continuous over $\mathbb{R}^{n}$.

The ratio $\frac{\phi}{\psi}$ is homogeneous in the bi-limit with triple $\left(\mathfrak{r}^{p}, \mathfrak{e}^{\phi, p}-\mathfrak{e}^{\psi, p}, \frac{\Phi^{p}}{\Psi^{p}}\right), p=0, \infty$.

\section{A. Designing homogeneous in the bi-limit functions from homogeneous functions}

In our paper we extensively use the following procedure for obtaining a homogeneous in the bi-limit function from the combination of two functions, the first one homogeneous in the $\infty$-limit and the other one homogeneous in the 0-limit.

\section{Proposition 1.8: Assume that}

(I) $\phi^{p}: \mathbb{R}^{n} \rightarrow \mathbb{R}, p=0, \infty$, is homogeneous in the p-limit with triple $\left(\mathfrak{r}^{p}, \mathfrak{v}^{p}, \Phi^{p}\right)$ and $\mathfrak{v}^{\infty}>0$ for all $k=1, \ldots, n$,

(II) $\phi^{\infty} \psi^{\infty}$ is homogeneous in the 0-limit with triple $\left(\mathfrak{r}^{0}, \mathfrak{v}^{0}, 0\right)$,

(III) $\phi^{0} \psi^{0}$ is bounded for all $x$

(IV) $\lim _{\|x\| \rightarrow 0} \psi^{0}(x)=\Psi^{0}, \lim _{\|x\| \rightarrow+\infty} \psi^{\infty}(x)=$ $\Psi^{\infty}$.

The function $x \mapsto \phi^{\infty}(x) \psi^{\infty}(x)+\phi^{0}(x) \psi^{0}(x)$ is homogeneous in the bi-limit with triple $\left(\mathfrak{r}^{p}, \mathfrak{v}^{p}, \Psi^{p} \Phi^{p}\right), p=0, \infty$.

B. Achieving sign definite homogeneous in the bi-limit functions

The following result shows how to render negative the difference $\xi-\gamma \sigma$, for some homogeneous functions $\xi, \sigma$ with the same degrees and with nonnegative $\sigma$, by a suitable choice of $\gamma>0$ and can be proved as the analogue result in [2].

Proposition 1.9: Assume that

(I) $\xi, \sigma: \mathbb{R}^{n} \rightarrow \mathbb{R}$ are homogeneous in the bi-limit with triple $\left(\mathfrak{r}^{p}, \mathfrak{v}, \Xi^{p}\right), p=0, \infty$, and, respectively, $\left(\mathfrak{r}^{p}, \mathfrak{v}, \Sigma^{p}\right), p=0, \infty$, with nonnegative $\sigma, \Sigma^{\infty}, \Sigma^{0}$, (II) $\left\{x \in \mathbb{R}^{n} \backslash\{0\}: \sigma(x)=0\right\} \subset\left\{x \in \mathbb{R}^{n} \backslash\{0\}\right.$ : $\xi(x)<0\}$,

(III) $\left\{x \in \mathbb{R}^{n} \backslash\{0\}: \Sigma^{p}(x)=0\right\} \subset\left\{x \in \mathbb{R}^{n} \backslash\{0\}\right.$ : $\left.\Xi^{p}(x)<0\right\}$.

There exists $\gamma^{*}>0$ such that

$$
(\xi-\gamma \sigma)(x)<0, \forall x \neq 0, \forall \gamma \geqslant \gamma^{*}
$$

and

$$
\left(\Xi^{p}-\gamma \Sigma^{p}\right)(x)<0, \forall x \neq 0, \forall \gamma \geqslant \gamma^{*}
$$

